\title{
Detection of Urethane in Non-alcoholic Fermented Food
}

\author{
Zhang Lihua ${ }^{1}$ \\ ${ }^{1}$ ChiFeng Indusrty Vocational Technology College, Inner Mongolia, 024005
}

\begin{abstract}
Among fermented foods, urethane often appears, which is a "2A" grade carcinogen. There are many types of fermented foods in my country, and the detection methods are not uniform enough, and no specific urethane detection standards have been formed. This article summarizes the specific experiments and results analysis based on previous work experience. At the same time, this article discusses six aspects from thin layer analysis, gas chromatography, gas chromatography-mass spectrometry, two-dimensional live multidimensional gas chromatography with stable isotope labeling mass spectrometry, high performance liquid chromatography-fluorescence, and nuclear magnetic resonance detection. The detection method of urethane in non-alcoholic fermented foods is introduced.
\end{abstract}

\section{Introduction}

With the continuous development of related science and technology, many chemicals have been fully applied in food production and processing. And under the effect of these raw materials, the main production efficiency has been greatly improved, which has brought higher economic benefits to related companies. But from another perspective, it will also bring harm to people who come into contact with these chemical raw materials. Urethane (EC) is also known as urethane, which is one of the common pollutants in the storage process of fermented matter. After fermentation, the related decomposition products are very likely to produce EC, which has a great impact on the human body. To this end, people need to do effective detection of EC in fermented food to maintain people's dietary health.

\section{Experimental Part}

\subsection{Main Instruments and Devices}

In the course of this experiment, the main equipment involved is the Clarus500 gas chromatography-mass spectrometer, which is produced by the American PE company and is equipped with an electron bombardment element. In addition, it will also be applied to rotary evaporators, ultrapure water devices, centrifuges, etc. These devices are all imported from abroad.

\subsection{Main Materials and Reagents}

The most common reagents are acetonitrile, methanol, nhexane, etc., and also include dichloromethane, ether, etc. These reagents are derived from the products of Sinopharm Group Chemical Reagent Co., Ltd. Throughout the entire standard of urethane, the specific purity is generally above $99 \%$. If it is D5-carbamate, the actual purity must be above $99.5 \%$.

\subsection{Standard Solution Configuration}

First of all, in the configuration of D5-EC stock solution, the first thing the experimenter needs to do is to weigh $0.01 \mathrm{~g}$ of D5-EC standard sample, and set the volume of methanol to $10 \mathrm{ml}$, the specific concentration is $1.00 \mathrm{~g} / \mathrm{L}$, and the optimal storage temperature is 0 to $4^{\circ} \mathrm{C}$. Second, the configuration of EC stock solution. The EC standard quality required for this type of configuration work is $0.01 \mathrm{~g}$, and it is also necessary to make the volume of methanol to $10 \mathrm{ml}$, the storage time is about three months, and the optimal temperature range is still 0 to $4{ }^{\circ} \mathrm{C}$. Once again, it is D5-EC use liquid configuration, the staff needs to accurately weigh $1.0 \mathrm{~g}$ D5-EC stock solution, with the help of methanol constant volume form, configure it to $2.00 \mathrm{mg} / \mathrm{L}$ solution, the actual constant volume is $50 \mathrm{ml}$, and the storage temperature is also 0 to $4^{\circ} \mathrm{C}$.

\subsection{Sample Preparation}

During the processing of vinegar and soy sauce samples, the experimenter can weigh $10.0 \mathrm{~g}$ of the sample and put it into a high-speed centrifuge tube, and add an appropriate amount of D5-carbamate and an appropriate amount of sodium chloride to it, so that It is saturated. Then add $10.0 \mathrm{ml}$ of acetonitrile, shake it for $1 \mathrm{~min}$, and let it stand for a period of time. After it is layered, perform two repeated extraction operations, and then combine the extracts together. For the study of the sauce sample, the experimenter also needs to weigh $10.0 \mathrm{~g}$ of the sample, add an appropriate amount of D5-carbamate and water, shake it evenly, add $10.0 \mathrm{ml}$ of acetonitrile to it, and take the acetonitrile phase after high-speed centrifugation. After repeating the extraction twice, the extracts were combined and concentrated according to actual requirements. 
Looking back at the tea beverage sample preparation, the same $10.0 \mathrm{~g}$ sample was added to the centrifuge tube, to which appropriate D5-carbamate and sodium chloride were added. When saturation occurs, add the same volume of methylene chloride as the overall solution. For the meat product sample, take $10.0 \mathrm{~g}$ into a high-speed centrifuge tube, add D5-carbamate to it, then add $10.0 \mathrm{ml}$ of acetonitrile to it, and finally concentrate to near dryness.

\subsection{Purification}

In order to present a better purification effect, the staff needs to add $2.0 \mathrm{ml}$ of acetonitrile to the concentration bottle. After the dissolution residue appeared, it was transferred to a $10 \mathrm{ml}$ centrifuge tube, and then $2 \mathrm{ml}$ of $\mathrm{n}$ hexane was added. After $1 \mathrm{~min}$ of vortex mixing, the staff can discard the n-hexane phase. After repeated purification operations, the staff must add $0.05 \mathrm{~g}$ of PSA filler in acetonitrile, and then perform the vortex mixing operation for $1 \mathrm{~min}$.

\subsection{Analysis Conditions}

First of all, it can be seen from the analysis of the chromatographic conditions that the most common content is the chromatographic column. In the actual temperature program, it is necessary to maintain a $1 \mathrm{~min} 50^{\circ} \mathrm{C}$ environment to avoid split injection problems. Looking back at the conditions of mass spectrometry, the main content involved is the electron bombardment of the ion source. The energy of such electrons can reach $70 \mathrm{eV}$, the actual ion source temperature is $230^{\circ} \mathrm{C}$, and the actual solvent delay time is $6 \mathrm{~min}$.

\section{Results and Analysis}

\subsection{Linear Relationship}

In the process of determining the actual linear relationship, the staff needs to accurately absorb the EC use solution to ensure that the standard solution concentration of the ECd5 internal standard meets the specific requirements. The common concentration coefficients are $0.05,0.10$ and 0.20 $\mathrm{mg} / \mathrm{L}$, etc. The specific measurement form is internal standard quantification method. If the measurement operation is performed under the same conditions, the experimenter can use the EC concentration as the abscissa, and use the ratio of the peak area of the corresponding concentration and the peak area of the internal standard as the ordinate to accurately draw the standard curve, as shown in Figure 1. From the information in the figure, it can be understood that when the EC is between 0.05 and $2.5 \mathrm{mg} / \mathrm{L}$, the linear correlation is the most lifeline, and the specific coefficient of correlation is $\mathrm{r}^{2}=0.9988$.

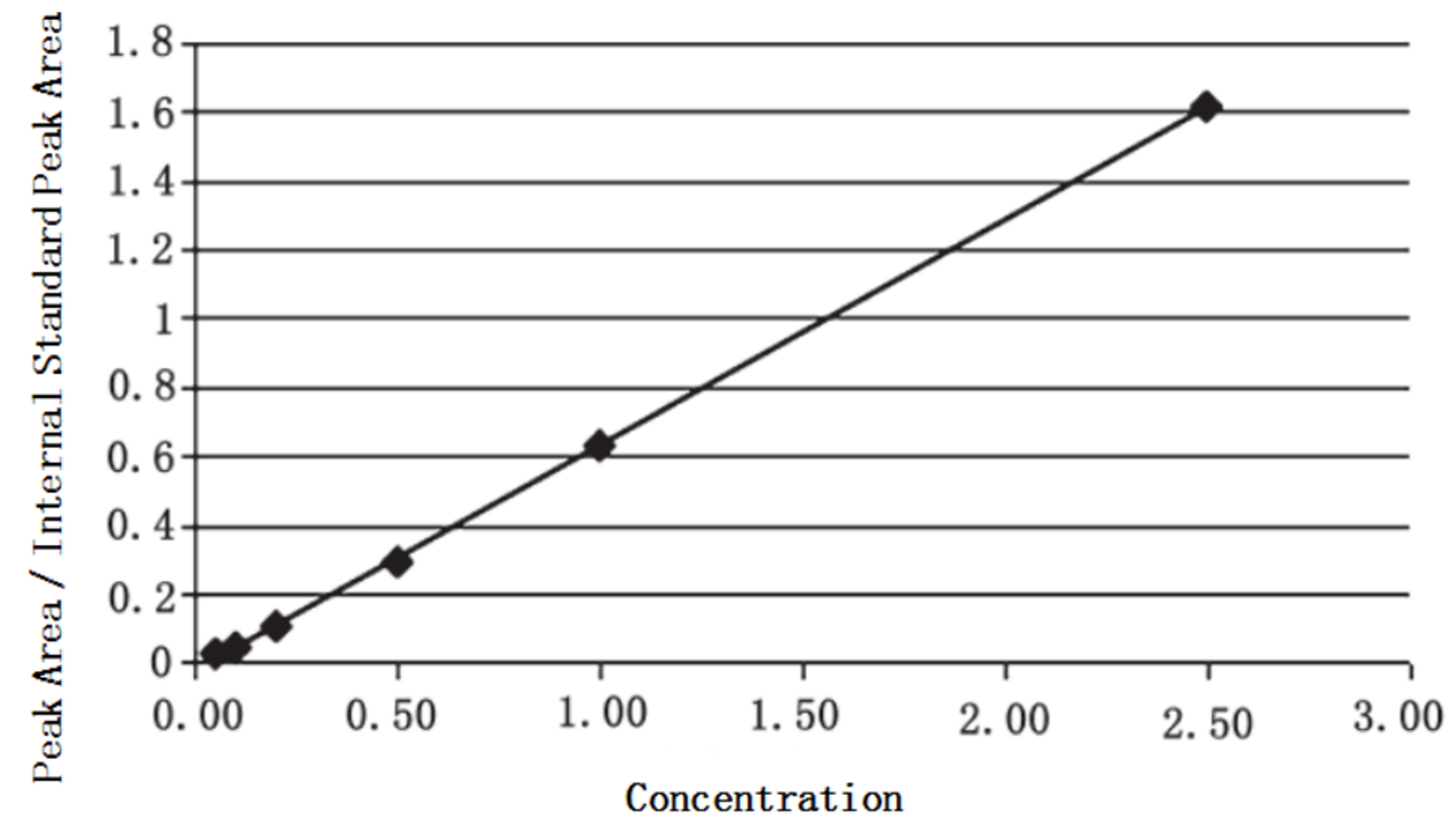

Figure 1 EC Standard Curve

\subsection{Limits of Detection, Limits of Quantitation, Recovery and Precision}

From the actual test execution process, it can be seen that for the EC samples that have not been found, it is also necessary to perform the measurement operation according to the above experimental steps to clarify the recovery rate, precision, detection limit and quantification limit. The confirmation of the spike level is mainly carried out at $0.02,0.05$ and $0.10 \mathrm{mg} / \mathrm{kg}$, and the actual number of parallel trials is about 6 times. For example, when calculating the EC detection limit with a 3 times signal-tonoise ratio, the final result is $0.005 \mathrm{mg} / \mathrm{kg}$. If it is 10 times the signal-to-noise ratio to calculate the EC detection limit, the final result is $0.01 \mathrm{mg} / \mathrm{kg}$. 


\section{Detection Method of Urethane in Non- alcoholic Fermented Food}

\subsection{Thin Layer Analysis}

It can be seen from the actual thin layer analysis method that the actual operation content is relatively simple, and the speed is relatively fast during the sample pretreatment and analysis process. The main content is mainly concentrated on the extraction of petroleum ether-nhexane, the specific ratio is $2: 1$, and the organic phase separation is carried out after the layer separation, and then is the combination process of the two extraction liquids, after drying shrinkage, benzene-di Methyl formamide benzene placed the concentrate on the chromatography plate. After drying for a period of time, the standard series of chromatography points showed blue-violet fluorescence at $\mathrm{R}=0.88$. Based on the above information, the experimenter can calculate the EC content in the sample. With this form, it can help the experimenter to perform semi-quantitative detection of EC in the liquid, but it is difficult to meet the national EC content standard detection requirements. Therefore, in order to better enhance the experimental effect, experimenters can use the flexibility of thin layer chromatography analysis to divide the separation process into manual, semi-automatic, and fully automatic states. At this time, TLC can also perform some other tasks while completing the task.

\subsection{Gas Chromatography}

In the application of meteorological chromatography, the mixture is mainly separated, and then the detection operation is performed on the single component, and it is precisely because of the execution of this type of operation that can help the staff to obtain corresponding qualitative and quantitative results. Throughout the entire application of gas chromatography, we will find that it has a great relationship with the analysis of organic and inorganic substances, its own thermal stability is extremely strong, the actual boiling point is concentrated below $500{ }^{\circ} \mathrm{C}$, and the molecular mass is below 400. The main targets are gas, Solids and liquids also make gas chromatography a specific separation and analysis tool, suitable for qualitative and quantitative analysis of EC. Secondly, as the analysis speed increases, an analysis cycle takes only a few minutes, and the application of an automated sample analysis system enhances its convenience features. Third, the sample is small and the sensitivity is high, which is suitable for application in ug-ng sample volume. Finally, this method exhibits extremely high efficiency, and can distinguish substances with similar substances, such as isotopes and isomers. But from the practical application point of view, gas chromatography itself has great limitations in the application process: first, if there is no standard sample for reference, it is difficult to confirm the unknown substance. Secondly, it is difficult to measure substances with higher boiling points by gas chromatography. Therefore, relevant staff need to have a comprehensive understanding of the advantages and disadvantages of gas chromatography. Only in this way can their effects be better presented.

\subsection{Gas Chromatography-mass Spectrometry}

In the application of this method, the most common forms are as follows: First, liquid extraction is mainly to distribute the two solvents in which the component to be detected and other impurities are incompatible with each other, to achieve better Separation and purification. Moreover, this operation method is relatively convenient, and does not require the preparation of special equipment, but the repeatability of the experiment is very poor, and emulsification is prone to occur, which further increases the detection error. However, this method can realize the optimization operation of the actual conditions, so that the selective pressurized liquid extraction method can be comprehensively improved. Studies have shown that the accuracy and precision of this method is better, the sample processing time is limited, it can be automated, and the detection of EC content becomes more effective. Second, the solid phase extraction method. When applying solid phase extraction abroad, the main purpose is to purify the $\mathrm{EC}$ in the liquid, and then use GC-MS qualitative and quantitative research to strengthen the accuracy of EC detection. From the analysis of the actual solid phase extraction column, it can be seen that the silica gel column will not adsorb the EC in the wine. In contrast, the CleSLE column can better show the EC adsorption effect and maintain the stability of the baseline. On this basis, the applicability of the technology itself is constantly improving. Third, the derivatization method. This method can achieve effective pretreatment of the sample. In the specific implementation process, an acidic medium is added to the derivatization reagent mixed sample solution, and then it is transferred to the syringe. The kinetics of the formation of different derivatives mainly include the stability of the derivatives to the reaction system, which improves the accuracy of the final detection results.

\subsection{Two-dimensional Live Multi-dimensional Gas Chromatography Coupled with Stable Isotope Labeling Mass Spectrometry}

The application of actual two-dimensional gas chromatography can not only show strong separation and analysis capabilities, but also simplify the pretreatment steps, strengthen the detection speed, and directly determine the EC content in fermented food. It is worth noting that the two-dimensional chromatography will have some restrictions on the sample, and the detection deviation will eventually be larger.

\subsection{High Performance Liquid Chromatography- fluorescence}

High-performance liquid chromatography-fluorescence method is mainly used in the detection of EC content of fermented foods. The n-hexane solution is used to extract the solid phase of the amino column. After that, the actual extract was eluted, and the extract form was separated by means of high-performance liquid chromatography and a 
fluorescence detector. The EC peak was extracted and compared with the peak area of the standard substance to obtain the EC concentration. Throughout the entire operation process, the actual steps are not complicated, the corresponding analysis time is short, and it can show strong practicability, but the accuracy corresponding to this method is limited, and the standard deviation is also obvious. Therefore, it is not suitable to apply this method in the implementation of some large sample screening operations.

\subsection{NMR Detection}

The highest NMR detection method is used to detect the EC content in fermented foods, which is mainly combined with the partial least squares method. The specific analysis time is 15 minutes each time, and the detection method under other methods is compared to prove the sensitivity and accuracy of the method. In addition, with the cooperation of related screening technologies, the analysis time of the instrument can be greatly shortened. In addition to the internal addition of buffer standard solution, it does not involve the preparation of other samples, nor does it require sample preparation. In general, nuclear magnetic resonance pop can support the determination of comprehensive structural information to determine whether EC exists in fermented food.

\section{Conclusion}

In summary, through the in-depth study of gas chromatography-mass spectrometry, experimenters can present the data of urethane in fermented food through the comparison of urethane content between different substances, so as to avoid the physical and mental health of the human being affected. However, it can also be seen from the actual research process that the traditional method has higher requirements for sample pre-processing procedures and the process is more complicated. For this reason, relevant experimenters must also rationally improve their detection methods.

\section{References}

1. Shu Zhigang, Chu Guoliang, An Kang. Research progress of urethane detection methods in beverage wine $[\mathrm{J}]$. Journal of Food Safety and Quality Inspection, 2019, 10(06): 1594-1600.

2. Zhou Jia, Luo Tingting, Cheng Changyu. The control strategy of urethane content during the production and storage of fermented bean curd $[\mathrm{J}]$. Journal of Food Safety and Quality Inspection, 2018, 9(16): 4280-4285.

3. Chen Rongfeng, Pan Yutian, Zhang Guoguang. Advances in detection methods of urethane in fermented foods[J]. Food Industry, 2018, 39(08): 263-268

4. Zheng Junke, Wang Shuang, Wei Xudong. Detection of urethane in non-alcoholic fermented foods [J]. Modern Food, 2018(13): 145-148.
5. Yao Xiaoruining, Zhou Xueyan, Xin Minhan. Screening of degradable urethane yeast and optimization of degradation conditions [J]. Science and Technology of Food Industry, 2018, 39(13): 155 161.

6. Zhao Yipeng, Wang Zongyi, Wang Guoying, Zhai Yongling. Stable isotope dilution-GC-MS/MS for rapid detection of ethyl carbamate in fermented food[J]. Chinese Journal of Food Science, 2018, 18(04): 306-311. 